\title{
A Laser Flash Photolysis Study of Chlorofluorocarbene
}

\author{
Eric M. Tippmann and Matthew S. Platz* \\ Department of Chemistry \\ 100 West $18^{\text {th }}$ Avenue \\ The Ohio State University \\ Columbus, Ohio 43210
}

\section{Submitted to the Journal of Physical Chemistry. Supporting Information:}

\section{List of Supporting Figures}

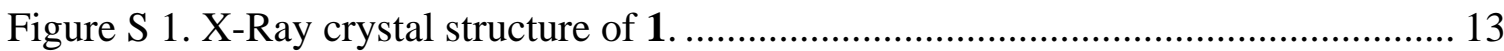

Figure $S 2$ A plot of $\mathrm{k}_{\text {obs }}$ of transient $\left(1142 \mathrm{~cm}^{-1}\right)$ decay as a function of [ $\left.\mathrm{LiBr}\right]$ in acetonitrile

Figure S 3. A plot of $\mathrm{k}_{\text {obs }}$ of transient $\left(1142 \mathrm{~cm}^{-1}\right)$ decay as a function of $\left[\mathrm{ZnBr}_{2}\right]$ in acetonitrile.

Figure S 4. UV/VIS spectrum of $\mathbf{1}$ (solid line) and $\mathbf{8}$ (dashed line) in cyclohexane. ...... 14 


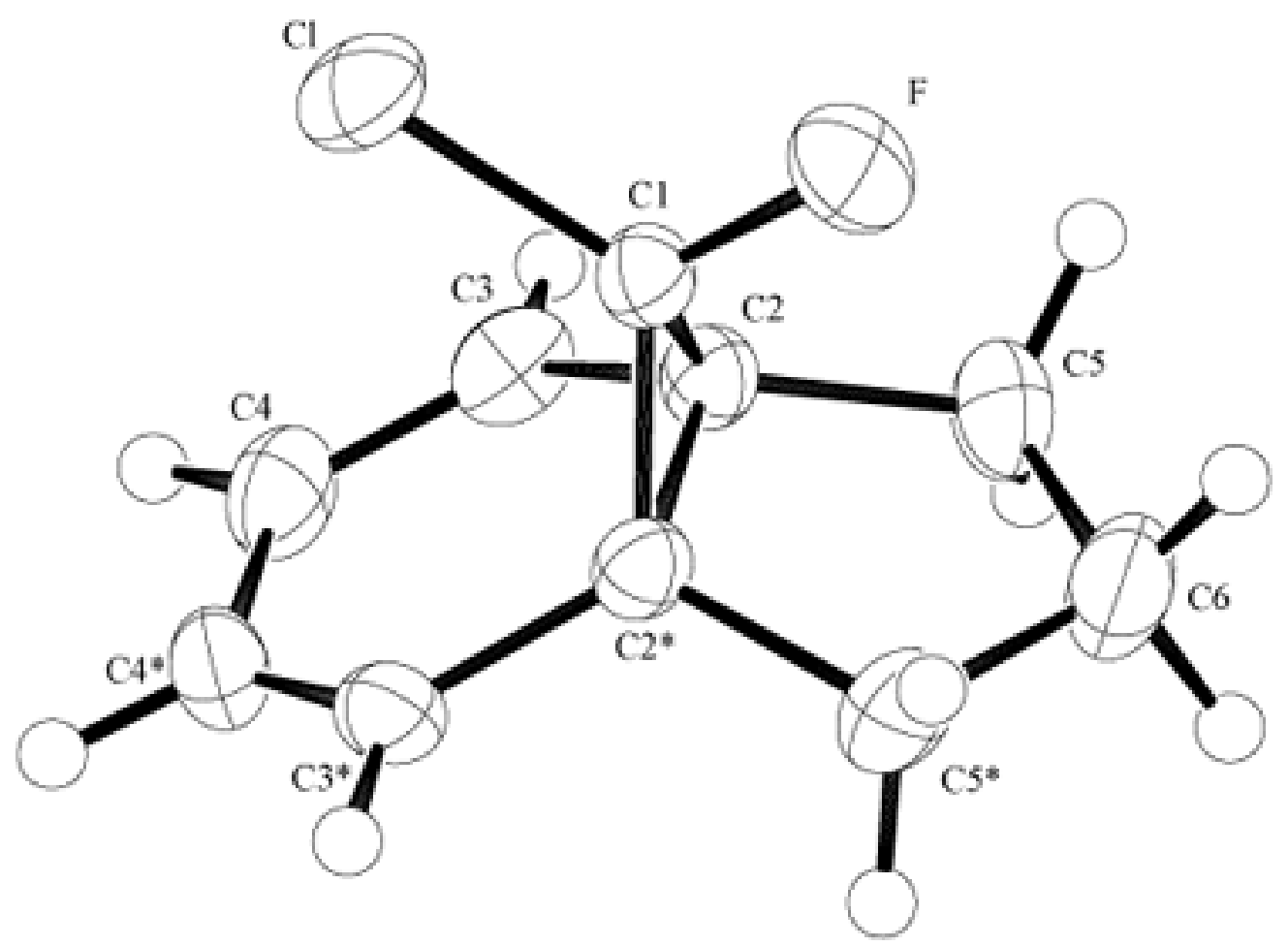

Figure S 1. X-Ray crystal structure of $\mathbf{1 .}$

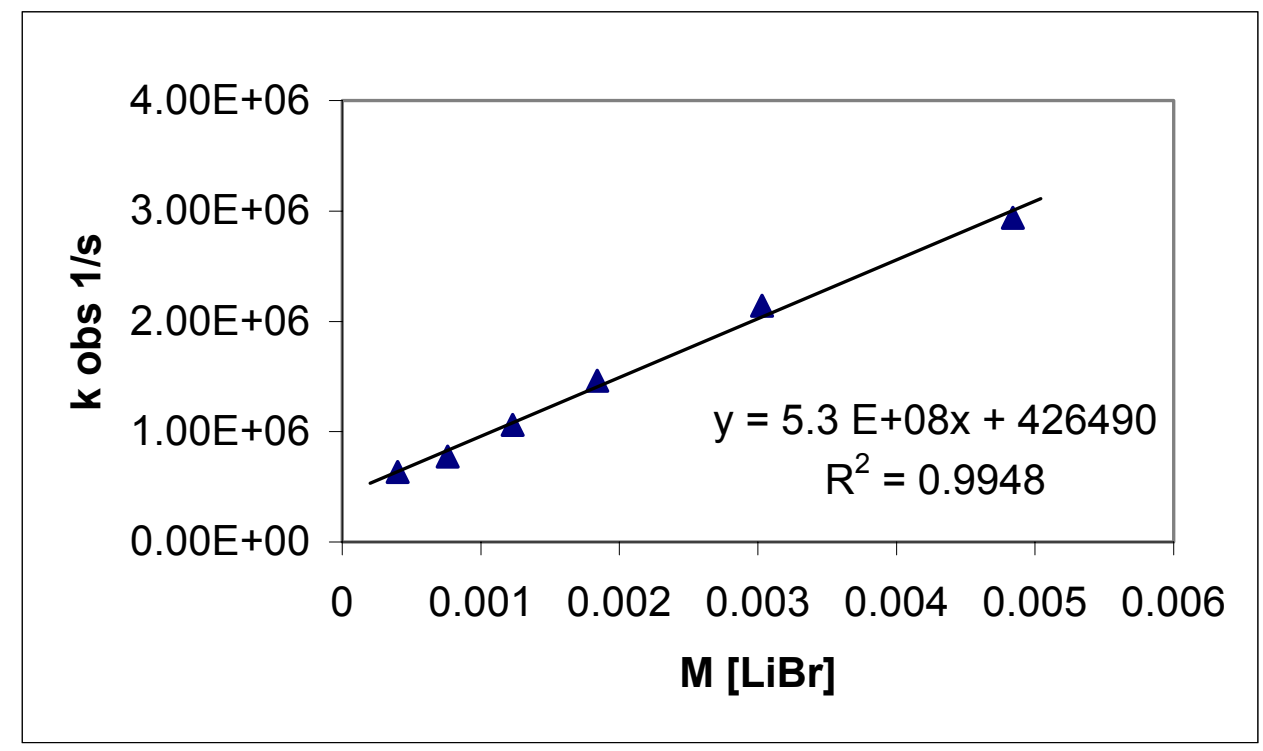

Figure S 2 A plot of $\mathrm{k}_{\mathrm{obs}}$ of transient $\left(1142 \mathrm{~cm}^{-1}\right)$ decay as a function of [LiBr] in acetonitrile. 


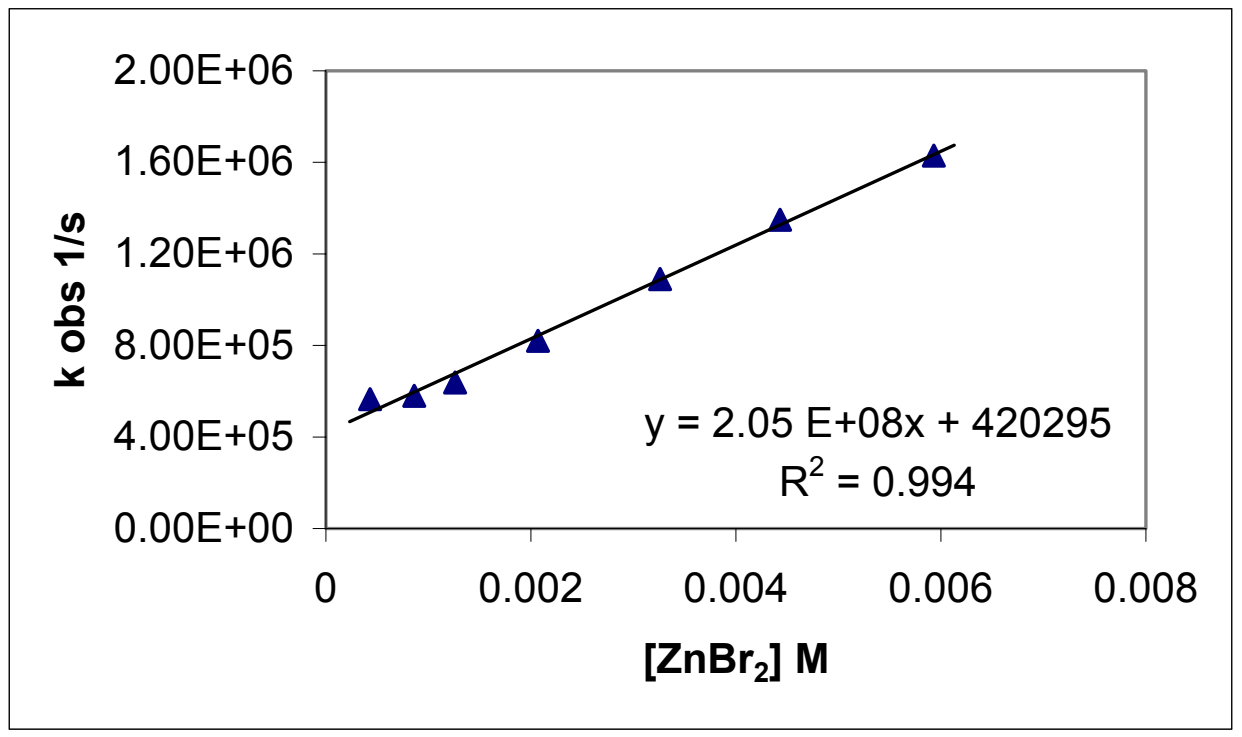

Figure S 3. A plot of $\mathrm{k}_{\mathrm{obs}}$ of transient $\left(1142 \mathrm{~cm}^{-1}\right)$ decay as a function of $\left[\mathrm{ZnBr}_{2}\right]$ in acetonitrile

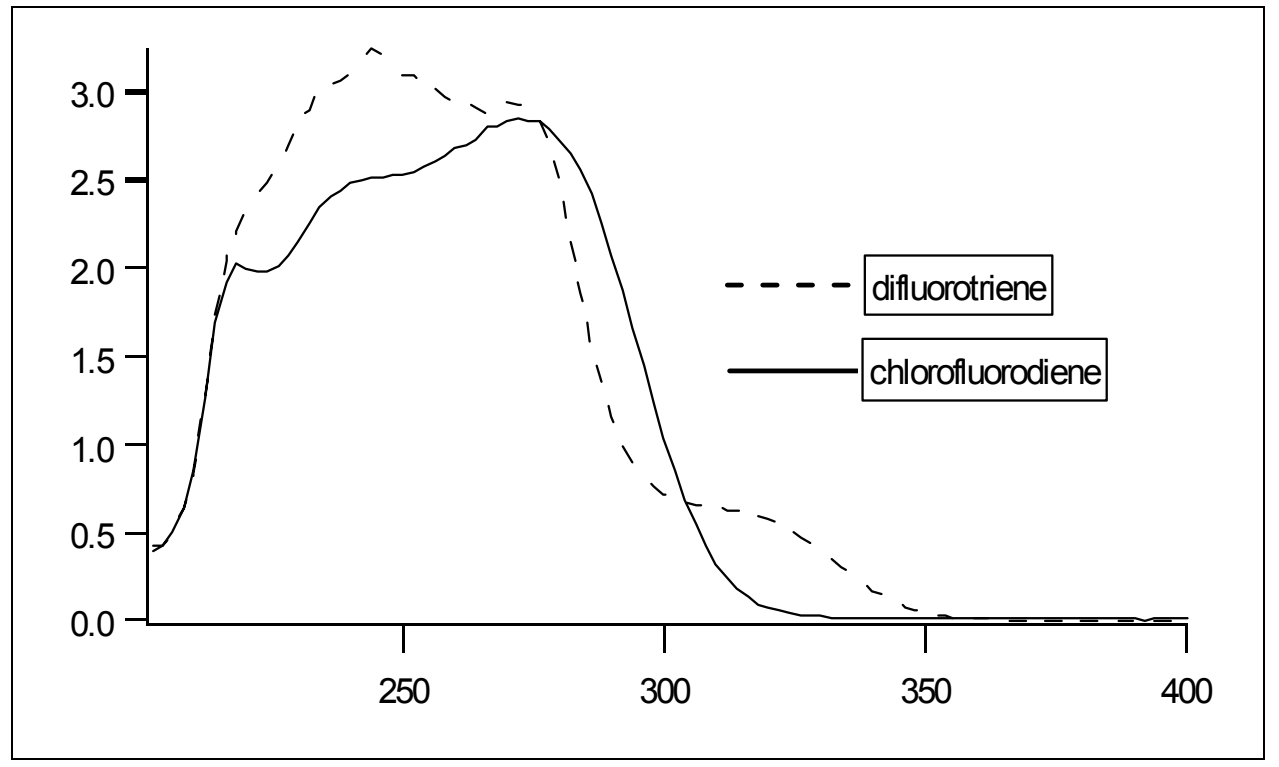

Figure S 4. UV/VIS spectrum of $\mathbf{1}$ (solid line) and $\mathbf{8}$ (dashed line) in cyclohexane.

\section{FCLBIRADICAL (5) COORDINATES}

$1 \backslash 1 \backslash$ GINC-ORIGIN $\backslash F O p t \backslash R B 3 L Y P \backslash 6-31 G(d) \backslash C 10 H 10 C 11 F 1 \backslash O S U 3075 \backslash 01-F e b-2003 \backslash 0$ \\# RB3LYP/6-31G* GEOM=(MODREDUNDANT) OPT FREQ \\fcldiene biradical 0131-03 frozen coordinates $\backslash \backslash 0,1 \backslash \mathrm{C},-2.2971748338,0.3485111235,-0.33973233$

$07 \backslash \mathrm{C},-2.3029800453,0.3281780119,1.0394300615 \backslash \mathrm{C},-1.1067017417,0.5572400$ 098,-1.0579715834\C,-1.0978880639,0.6229105093,1.7618230211\C,0.085396 
$5977,0.776591081,1.1172867414 \backslash \mathrm{C}, 0.2032015337,0.5638058273,-0.365729202$ $3 \backslash \mathrm{C}, 0.5004519053,-0.9256846534,-0.8171616114 \backslash \mathrm{C}, 1.3726003575,1.47247291$ $41,-0.7843459878 \backslash \mathrm{C}, 2.3313334906,1.3849838363,0.421512883 \backslash \mathrm{C}, 1.408861406$ $4,1.2702597541,1.6653728576 \backslash \mathrm{Cl},-0.2282274385,-2.3078300268,-0.04407005$ $32 \backslash \mathrm{F}, 0.6814287761,-1.1240824684,-2.1378300404 \backslash \mathrm{H},-3.2244528361,0.215311$ $9167,-0.8911843904 \backslash \mathrm{H},-3.2266398592,0.1633616008,1.5855373529 \backslash \mathrm{H},-1.1107$ $285125,0.5806835358,-2.1423778812 \backslash \mathrm{H},-1.1559717717,0.7840929957,2.83682$ $28881 \backslash \mathrm{H}, 1.831731277,1.1695343469,-1.7297530795 \backslash \mathrm{H}, 0.9860568648,2.492576$ $9649,-0.9040683256 \backslash \mathrm{H}, 2.9459682574,0.4831899098,0.3314607879 \backslash \mathrm{H}, 3.011719$ $9314,2.2399612827,0.4772334469 \backslash \mathrm{H}, 1.2832857626,2.2397583566,2.166260967$ $2 \backslash \mathrm{H}, 1.8234347164,0.5857712786,2.4168204067 \backslash \backslash$ Version=SGI64-G98RevA.9\HF $=-947.0003495 \backslash \mathrm{RMSD}=9.550 \mathrm{e}-09 \backslash \mathrm{RMSF}=1.908 \mathrm{e}-03 \backslash \mathrm{Dipole}=0.0015287,0.6745165$ ,0.3032802 $\backslash \mathrm{PG}=\mathrm{C} 01[\mathrm{X}(\mathrm{C} 10 \mathrm{H} 10 \mathrm{Cl1F} 1)] \backslash \backslash$ @

Frequencies

$\begin{array}{lcc}-362.9082 & 88.5129 & 101.310 \\ 5126.7859 & 151.1420 & 220.4457 \\ 272.6998 & 299.5035 & 326.8963 \\ 389.3250 & 427.3596 & 503.7196 \\ 513.5479 & 532.0533 & 563.8640 \\ 611.9905 & 654.0652 & 720.9184 \\ 747.6851 & 820.5870 & 846.9595 \\ 861.3280 & 888.2386 & 920.7907 \\ 925.6686 & 945.6923 & 966.8759 \\ 1015.6954 & 1038.3808 & 1069.1265 \\ 1078.7411 & 1149.8197 & 1157.9010 \\ 1163.0302 & 1179.4091 & 1191.0145 \\ 1210.3268 & 1252.5535 & 1275.4915 \\ 1311.7745 & 1341.0919 & 1360.1494 \\ 1369.0388 & 1420.6660 & 1492.6084 \\ 1504.2664 & 1516.8819 & 1533.6328 \\ 1554.3216 & 1684.9760 & 3036.2275 \\ 3058.0169 & 3062.3040 & 3078.2787 \\ 3117.9523 & 3125.7801 & 3172.7041 \\ 3186.8521 & 3212.3393 & 3220.8071\end{array}$

Fclcarbene (1) coordinates

$1 \backslash 1 \backslash$ GINC-ORIGIN $\backslash$ Freq $\backslash R B 3 L Y P \backslash 6-31 G(d) \backslash C 1 C 11 F 1 \backslash O S U 3075 \backslash 20-J a n-2003 \backslash 0 \backslash \ N$ GEOM=ALLCHECK GUESS=TCHECK RB3LYP/6-31G(D)

FREQ $\backslash$ FClcarbene $\backslash \backslash 0,1 \backslash C,-0.6323827612,0.6706985268,0 . \mid F,-1.467965797,-$ $0.3249804285,0 . \mid \mathrm{Cl}, 1.0003522788,-0.064668665,0.1 \backslash$

Version $=$ SGI64-G98RevA.9\State $=1-\mathrm{A}^{\prime} \backslash \mathrm{HF}=-598.034$ $0669 \backslash \mathrm{RMSD}=9.852 \mathrm{e}-10 \backslash \mathrm{RMSF}=3.612 \mathrm{e}-05 \backslash \mathrm{Dipole}=-0.1454239,-0.2540567,0 . \backslash \mathrm{Dip}$ oleDeriv $=1.896417,-0.0943142,0.0001766,0.3997399,0.7683508,0.0000666,0$ $.0000026,0.0001517,-0.1417247,-0.8196852,-0.2214085,-0.0000635,-0.9696$ $622,-0.4726321,-0.0001018,-0.0001058,-0.0000502,0.1250697,-1.0767319,0$ $.3157228,-0.0001131,0.5699224,-0.2957188,0.0000351,0.0001031,-0.000101$ 
$5,0.0166549 \backslash$ Polar $=33.38099,-4.7679599,16.2048745,-0.0024604,-0.0002069$ $, 13.0968091 \backslash \mathrm{PG}=\mathrm{CS}[\mathrm{SG}(\mathrm{C} 1 \mathrm{Cl1F} 1)] \mathrm{NImag}=0 \backslash \backslash 0.24076774,0.09347359,0.37421$ $541,0.00002281,0.00000554,-0.00012800,-0.16930855,-0.15956892,-0.00000$ $263,0.24066548,-0.15124013,-0.29221394,-0.00001018,0.18120222,0.257662$ $38,-0.00000982,-0.00000298,0.00017544,0.00000161,0.00000395,-0.0001402$ $6,-0.07145918,0.06609533,-0.00002018,-0.07135693,-0.02996209,0.0000082$ $1,0.14281611,0.05776654,-0.08200146,0.00000463,-0.02163330,0.03455157$, $-0.00000097,-0.03613323,0.04744989,-0.00001300,-0.00000256,-0.00004745$ $, 0.00000102,0.00000623,-0.00003517,0.00001197,-0.00000366,0.00008262 \backslash \backslash$ $-0.00002286,-0.00008232,0 ., 0.00001698,0.00006028,0 ., 0.00000588,0.00002$ 204,0.11@

frequencies

$\begin{array}{lll}426.2524 & 727.4672 & 1216.4394\end{array}$

chlorofluorodiene (2)

$\mathrm{N}-\mathrm{N}=8.285446823168 \mathrm{D}+02 \mathrm{E}-\mathrm{N}=-3.884527187410 \mathrm{D}+03 \mathrm{KE}=9.412216946407 \mathrm{D}+02$ $1 \backslash 1 \backslash$ GINC-ORIGIN\FOpt $\backslash R B 3 L Y P \backslash 6-31 \mathrm{G}(\mathrm{d}) \backslash \mathrm{C} 10 \mathrm{H} 10 \mathrm{Cl1F} \backslash \mathrm{OSU} 3075 \backslash 14-J a n-2003 \backslash 0$ \\\#N B3LYP/6-31G* OPT FREQ $\backslash \backslash$ fcl diene precursor $\backslash \backslash 0,1 \backslash \mathrm{C},-1.2713618016,-$ $1.0089050752,1.0898263109 \backslash \mathrm{C},-2.3992147576,-0.4449728091,0.6094769746 \backslash \mathrm{C}$ $, 0.0381721063,-0.7771862107,0.4477877118 \backslash \mathrm{C}, 0.0225705313,0.064680558,-0$ $.8951517427 \backslash \mathrm{C},-2.4135595372,0.3294948073,-0.6260409904 \backslash \mathrm{C},-1.2998171361$ $, 0.5277759127,-1.3616179212 \backslash \mathrm{C}, 0.6397113991,0.5925905842,0.3640548834 \backslash \mathrm{C}$ ,1.0260689664,-1.9326905489,0.2596116136\C,1.8065874093,-1.6503650492, $-1.0555105859 \backslash \mathrm{C}, 1.0014226531,-0.5979262938,-1.8695140928 \backslash \mathrm{Cl},-0.0326796$ $458,1.9801969734,1.241752062 \backslash \mathrm{F}, 2.0069906357,0.6732183324,0.3986767704 \backslash$ $\mathrm{H},-1.3073044784,-1.6951147186,1.9331728966 \backslash \mathrm{H},-3.3483445425,-0.63048827$ $41,1.1056503024 \backslash \mathrm{H},-3.3727182192,0.6852050384,-0.9933784272 \backslash \mathrm{H},-1.356981$ $0433,0.9872108011,-2.3460257727 \backslash \mathrm{H}, 0.4445204791,-2.8580861601,0.1788824$ $798 \backslash \mathrm{H}, 1.7048865576,-2.0470369406,1.1122340371 \backslash \mathrm{H}, 2.7970015985,-1.256918$ $0704,-0.8203364875 \backslash \mathrm{H}, 1.9515461472,-2.5688749881,-1.6329998976 \backslash \mathrm{H}, 1.6647$ $838361,0.1267000831,-2.3549992738 \backslash \mathrm{H}, 0.4117689245,-1.0798855617,-2.6576$ 088146 $\backslash$ Version=SGI64-G98RevA.9\HF=-947.0525172 $\backslash \mathrm{RMSD}=7.535 \mathrm{e}-09 \backslash \mathrm{RMSF}=5$. $520 \mathrm{e}-05 \backslash$ Dipole $=-0.3048812,-0.8297105,-0.5165689 \backslash \mathrm{PG}=\mathrm{C} 01[\mathrm{X}(\mathrm{C} 10 \mathrm{H} 10 \mathrm{Cl1F} 1)$ ]11@ frequencies 141.8378 184.6527

313.7729 429.6463

537.1105

673.1540

779.9608

898.7495

959.5312

997.2296

1113.2330

$\begin{array}{cc}151.9832 & 180.6198 \\ 209.6002 & 232.6636 \\ 362.5819 & 392.5293 \\ 488.4876 & 509.8390 \\ 592.0790 & 641.1638 \\ 676.8123 & 751.3599 \\ 809.2322 & 813.4289 \\ 900.4515 & 947.7371 \\ 972.3490 & 987.3400 \\ 1029.4708 & 1081.3925 \\ 1115.8049 & 1193.8099\end{array}$




$\begin{array}{lll}1197.4201 & 1210.1275 & 1216.4375 \\ 1220.4630 & 1266.8256 & 1274.5190 \\ 1322.6336 & 1348.6468 & 1360.5030 \\ 1421.9211 & 1427.1614 & 1473.1140 \\ 1519.5194 & 1520.5697 & 1545.4840 \\ 1623.4947 & 1706.1744 & 3060.4684 \\ 3060.9431 & 3088.1963 & 3097.0762 \\ 3100.5966 & 3142.7433 & 3172.9423 \\ 3177.0024 & 3192.8237 & 3204.7712\end{array}$

chlorofluorotriene (6) coordinates

$1 \backslash 1 \backslash$ GINC-ORIGIN $\backslash F O p t \backslash R B 3 L Y P \backslash 6-31 G(d) \backslash C 10 H 10 C 11 F 1 \backslash O S U 3075 \backslash 17-J a n-2003 \backslash 0$

॥\# RB3LYP/6-31G* OPT=MODREDUNDANT FREQ=NORAMAN $\backslash \backslash$ dfluorotriene note modredundant $\backslash \backslash 0,1 \backslash \mathrm{C},-1.7171395572,-1.0189764336,-1.310834392 \backslash \mathrm{C},-1.716482$ $3455,-0.872288917,0.0962709888 \backslash \mathrm{C},-0.5587022149,-1.0188895795,-2.086836$ $0839 \backslash \mathrm{C},-0.7397810585,-0.2047522701,0.8092135716 \backslash \mathrm{C}, 0.7422826575,-0.8721$ $049459,-1.5507803157 \backslash \mathrm{C}, 1.0298634996,-0.2046204969,-0.3762168582 \backslash \mathrm{C}, 2.02$ $28082446,-0.6662786033,0.6728741118 \backslash \mathrm{C}, 0.1123102401,0.8317884065,0.1677$ $522141 \backslash \mathrm{C}, 1.2011123078,-1.3890752352,1.7929014841 \backslash \mathrm{C},-0.1474047385,-0.66$ $64376709,2.1266336411 \backslash \mathrm{Cl},-0.6567132841,1.9907185744,-0.9801384761 \backslash \mathrm{F}, 0$. $7418146492,1.6259839507,1.1075852908 \backslash \mathrm{H},-2.6282210057,-1.3823034713,-1$. $7815548332 \backslash \mathrm{H},-2.4371050318,-1.4636867474,0.660165721 \backslash \mathrm{H},-0.6472845929$,$1.3821550684,-3.1085234147 \backslash \mathrm{H}, 1.538031585,-1.4633894734,-2.0026563622 \backslash \mathrm{H}$ ,2.5829240657,0.1738349003,1.0928943641 \H,2.7387876133,-1.3701419324,0 $.2360043024 \backslash \mathrm{H}, 0.9551604686,-2.3926900973,1.425628184 \backslash \mathrm{H}, 1.8082881327,-1$ $.5089708766,2.6992977224 \backslash \mathrm{H}, 0.0277956029,0.1736485692,2.8044961858 \backslash \mathrm{H},-0$ $.8237850601,-1.3704026488,2.622464437 \backslash \backslash$ Version$=S G I 64-G 98 R e v A .9 \backslash H F=-947$ $.0507094 \backslash \mathrm{RMSD}=8.401 \mathrm{e}-09 \backslash \mathrm{RMSF}=6.964 \mathrm{e}-04 \backslash \mathrm{Dipole}=0.2357349,-1.244202,0.35$ 17729\PG=C01 [X(C10H10C11F1)] @

frequencies

$\begin{array}{lcl}157.8590 & 172.4410 & 183.1081 \\ 218.8424 & 260.3028 & 315.2569 \\ 333.9826 & 380.9798 & 406.9310 \\ 416.9947 & 434.3312 & 464.2413 \\ 560.9386 & 565.2848 & 599.2092 \\ 659.0922 & 659.6100 & 760.1272 \\ 814.5772 & 815.9571 & 839.3765 \\ 843.0496 & 860.5623 & 911.4965 \\ 964.3555 & 979.8606 & 983.0195 \\ 997.0397 & 1021.4199 & 1086.6815 \\ 1100.6254 & 1117.1761 & 1165.6576 \\ 1195.2163 & 1199.2904 & 1219.9255 \\ 1246.8812 & 1253.6043 & 1338.5492 \\ 1353.1179 & 1360.6642 & 1375.1809 \\ 1404.5452 & 1482.0544 & 1491.0835 \\ 1509.1997 & 1523.0846 & 1533.5401\end{array}$


$\begin{array}{lll}1606.0541 & 1630.1649 & 3048.4715\end{array}$

$3078.6015 \quad 3078.8653 \quad 3091.8766$

$\begin{array}{lll}3125.7529 & 3130.3153 & 3156.6848\end{array}$

$\begin{array}{lll}3158.9387 & 3172.9974 & 3189.7098\end{array}$ 